\title{
Vaccine development strategies for improving immunization: the role of modern immunology
}

\author{
Myron M Levine \& Marcelo B Sztein
}

\author{
An ideal vaccine has certain biological and physical characteristics. Technological advances have provided new \\ strategies that may help the design of such a vaccine.
}

$\bigvee_{\text {for }}^{\text {accin }}$ accines constitute cost-effective measures for preventing disease. Epidemiologically targeted implementation of vaccines has diminished morbidity and mortality from infectious diseases that previously were scourges and economic burdens (such as measles, polio, diphtheria, invasive Haemophilus influenzae type b and pneumococcal infections). Global programs have eradicated smallpox and reduced poliomyelitis transmission such that eradication is feasible. Advances in biotechnology and an understanding of the inductive and effector components of immune responses have ushered in a 'golden age' of vaccine development and implementation. Nevertheless, other factors, some concocted by less thoughtful humans, have also changed the course of vaccine development or usage ${ }^{1}$ After the deliberate release of anthrax spores by bioterrorists in the US in 2001, resources were diverted to develop vaccines against potential bioterror agents (mainly pathogens such as Bacillus anthracis, Yersinia pestis and Francisella tularensis or toxins such as botulinum toxins that can be spread by aerosolization). The emergence of new pathogens such as severe acute respiratory syndrome virus and the aggressive dissemination of recognized pathogens such as West Nile virus also stimulate vaccine development programs.

This is a propitious moment to examine the landscape of vaccine development and immunization from a global perspective and to consider how burgeoning immunological knowledge and biotechnological advances are being harnessed. This com-

Myron M. Levine and Marcelo B. Sztein are in the Center for Vaccine Development, University of Maryland School of Medicine, Baltimore, Maryland 21201, USA.

e-mail: mlevine@medicine.umaryland.edu mentary on preventive vaccines against infectious agents identifies the desirable characteristics a vaccine should have and discusses strategies to achieve them.

\section{Characteristics of ideal vaccines}

Many licensed vaccines have one or more ideal characteristics (Box 1), but none manifests them all. Although vaccine safety is an issue worldwide, this concern is particularly conspicuous in industrialized countries where the very success of vaccines has led the public to forget the dangers of previously common infectious diseases and instead to dwell on rare adverse events attributed to vaccines. Some adverse events are indeed vaccine associated, whereas for others there is no valid basis for 'incriminating' vaccines. For example, in the US and many European countries, inactivated poliovirus vaccine replaced live oral Sabin polio vaccine because in rare cases the latter caused vaccine-associated paralytic poliomyelitis. Similarly, acellular pertussis vaccine has supplanted whole-cell pertussis vaccine, which causes high fever in some infants. In contrast, neither biological nor epidemiological evidence incontrovertibly supports the contention that measlesmumps-rubella vaccine precipitates autism or inflammatory bowel disease, even rarely. The challenge faced in developing new vaccines is to achieve strong immunogenicity without increasing 'reactogenicity'. In developing countries, where infectious diseases morbidity and mortality burden remains high, a different risk-benefit ratio prevails. In such venues, generally mild untoward effects and serious but rare adverse events attributable to vaccines (such as vaccine-associated paralytic poliomyelitis) are considered an acceptable price for the prevention of death and debilitating disease for the masses.

Another chief consideration for vaccines is that they confer long-lived efficacy, an important determinant of cost-effectiveness after implementation. Some wild-type infections (measles) and vaccines (17D yellow fever) confer enduring, even lifelong, immunity after a single immunizing event. Key to the development of vaccines that elicit enduring protection is the induction of strong, long-lived immunological $\mathrm{T}$ and $\mathrm{B}$ cell memory to antigens that correlate with protection; that is, the ability to 'recall' previous exposures to antigen and to mount enhanced, accelerated effector responses ${ }^{2-5}$. Research in nonhuman primates and in humans using new immunological and flow cytometry techniques is identifying the cells responsible for maintaining $\mathrm{T}$ and $\mathrm{B}$ cell memory and long-lived protection after vaccination. Future measurements of the specificity, subsets, magnitude and longevity of $\mathrm{T}$ and $\mathrm{B}$ memory responses elicited by immunization may guide vaccine development by providing immunological correlates of longlived protection before epidemiological data become available.

Ideally, just a single dose of vaccine should confer robust, long-lived immunity. This characteristic is especially relevant in developing countries, where populations may not have easy access to immunization services. So far, only a few live vaccines have achieved this goal. In practice, even some of the most successful live virus vaccines (measles and rubella) are administered on a second occasion, as before school entry, to immunize subjects who failed to immunoconvert after the first dose and to boost the immunity of those who did respond initially. In contrast to the results with live vaccines, it has been difficult to immunize with a single dose of nonliving antigen vaccines. One goal of modern vaccine development is to rectify this using new adjuvants (such as MF59 and cytidine phosphate guanosine (CPG)) and antigen delivery systems (such as proteasomes and virus-like particles). 


\section{BOX 1 SOME CHARACTERISTICS OF AN IDEAL VACCINE}

- Shows an impeccable safety profile in all populations, including young infants, the elderly and immunocompromised subjects (such as HIV-positive subjects)

- Elicits a high level of long-lived efficacy, including in young infants and the elderly

- Requires only a single dose (or at most two doses spaced fairly close together) to confer protection

- Stimulates protection within 2 weeks of administration

- Administrable without a needle and syringe; that is, orally, nasally or transcutaneously or with a needle-free injection device

- Administrable in combination with (in the same formulation) or concomitantly (coadministered) with other vaccines

- Can be manufactured in large scale and with quality control by relatively uncomplicated and economical processes

- Amenable to production in formulations that are resistant to high and low temperatures and therefore free from strict storage requirements

Vaccine efficacy depends on the immune responses of individuals. Thus, immunizing very young infants, the elderly and immunocompromised persons poses a challenge. In industrialized countries, where people live longer and an increasing proportion of the population is elderly, public health immunization programs are progressively targeting the elderly. However, senescence of the immune system in the elderly blunts immune responses to many vaccines. In developing countries, primary infant immunizations are typically administered at 6,10 and 14 weeks of age to stimulate protection against pertussis and other infections that pose risks early in infancy. However, young infants are difficult to immunize because of immunological immaturity and the blocking effect of maternal antibodies ${ }^{6}$. Vaccines that have greater immunogenicity and powerful yet safe adjuvants are needed to successfully immunize the very young and the elderly. For immunocompromised subjects, less immunogenicity is generally acceptable, as long as the vaccine is safe.

Ideally, vaccines should be administrable without needles and syringes (orally, nasally or transcutaneously, or with needle-free injection devices ${ }^{7}$ ). In developing countries, injection safety is a formidable problem, as in some venues nonsterile needles and syringes are incorrectly reused to administer vaccines, sometimes spreading hepatitis B and C and HIV. Even when this problem has been diminished through the use of single-use 'auto-disable' syringes, safe disposal of the infectious waste (used syringes and needles) remains a daunting challenge. The world would indeed be better immunized if all vaccines could be administered with the simplicity of the Sabin live oral polio vaccine, which requires merely the deposition of a few drops into the subject's mouth.
Another trait of an ideal vaccine is its feasibility to be administered in combination with other vaccines. This allows fewer healthcare visits, minimizes inconvenience and trauma and diminishes risks of needle sticks. Any contact with health workers for the purpose of vaccination should be an opportunity to administer multiple vaccines. This is particularly true in developing countries in which populations may have limited access to immunization services. Combination formulations are particularly desirable so that infants are not given multiple injections at the same visit. Clinical trials of some candidate combination vaccines for infants have shown that the immunological response to one component of a combination was significantly diminished or enhanced compared with the response to the same antigen inoculated separately. Frustratingly, preclinical immunogenicity studies did not predict these imbalanced immune responses. Combination vaccines generally fare better when administered mucosally, as the vast mucosal surface with its many inductive sites is more 'forgiving' in allowing several vaccines or serotypes to be delivered in combination without loss of immunogenicity.

Ideally, vaccines should have uncomplicated, economical large-scale manufacture processes, because simplicity of manufacture has long-term implications for vaccine supply and cost. Vaccines differ enormously in their complexity and ease of manufacture. Some complex vaccines (such as multivalent pneumococcal conjugate) are challenging to manufacture. Others (such as attenuated Salmonella enterica serovar Typhi (S. Typhi) vaccine strain Ty21a are relatively simple to manufacture.

An ideal vaccine would be formulated to resist high and low temperatures, to facili- tate distribution. In developing countries, the World Health Organization-UNICEF Expanded Program on Immunization assumes the formidable task of delivering multiple vaccines to populations at risk (mainly infants and pregnant women). Routine immunization is provided through fixed health centers and mobile outreach services complemented (for certain vaccines like polio and measles) by periodic mass campaigns. The Expanded Program on Immunization invests enormous financial and human resources to maintain a 'cold chain' to keep vaccines within a restricted temperature range lest potency be damaged by excessive heat (live viruses) or freezing (protein vaccines). 'Glassification' technologies that dry vaccines in the presence of sugars such as trehalose or other stabilizers render vaccines resistant to high and low temperatures. Should clinical trials of these products show seroequivalence to formulations now licensed, this technology could relieve pressures on the 'cold chain' in developing countries.

\section{Vaccine technologies and strategies}

Of the generic vaccine technologies and vaccination strategies in different stages of development, some have already demonstrated their flexibility, practicality, robustness and potential simplicity of production and others hold promise for the future (Box 2; this list is illustrative, not comprehensive). These strategies address the desired characteristics of an ideal vaccine in various ways (Table $\mathbf{1}$ ).

Conjugate vaccines consist of poorly immunogenic $\mathrm{T}$ cell-independent antigens or haptens (polysaccharides and peptides) covalently linked to highly immunogenic carrier proteins (such as tetanus and diphtheria toxoids). Linkage to the carrier protein converts the $\mathrm{T}$ cell-independent antigen to a $\mathrm{T}$ cell-dependent antigen that elicits immunological memory. Licensed conjugate vaccines against $H$. influenzae type b, pneumococcus and meningococcus are safe and highly immunogenic in young infants. This generic vaccine strategy has an exceptional track record of safety and effectiveness and is relevant for any defined antigen intended to elicit serum antibodies. Moreover, serum antibodies stimulated by conjugate vaccines apparently transude onto mucosal surfaces, where they interfere with colonization of respiratory (and perhaps gastrointestinal) pathogens and foster herd immunity by diminishing transmission.

Live vaccines tend to be more immunogenic than nonliving antigens because they can proliferate and elicit strong innate and 
adaptive responses. Many successful live viral and bacterial vaccines, such as attenuated measles virus, poliovirus, rubella virus and S. Typhi strain Ty21a, were produced by repetitive in vitro passage or by nonspecific mutagenesis. Now precise deletion mutations can be introduced into wild-type organisms, resulting in rational attenuation. Drawbacks to live vaccines are their potential transmissibility and reactogenicity in immunocompromised hosts.

Two categories of bacteria serve as live vector vaccines to express protective antigens of unrelated pathogens and to deliver them to the immune system: normal flora commensals and attenuated derivatives of pathogens. Commensal flora of the human gastrointestinal or respiratory tract, such as Lactobacillus, Lactococcus or Streptococcus gordonii, are well tolerated and are able to colonize. However, because commensals manifest few 'danger signals' to stimulate the innate immune system, they tend to be modestly immunogenic. Among the most popular attenuated strains of known pathogens used as live vectors are derivatives of $S$. Typhi and shigella. The attractive qualities of $S$. Typhi and shigella include their ability to be administered mucosally and the broad humoral (systemic and mucosal) and cell-mediated immune responses they generate. For bacterial live vectors to elicit strong responses to foreign antigens they carry, genes encoding the guest antigen must be stabilized, foreign gene expression should be delayed until the live vector attains an appropriate niche within the host and the foreign antigen should be secreted or expressed on the surface of the live vector. Bacterial live vectors carrying either prokaryotic expression systems or eukaryotic expression systems (that is, DNA vaccines) encoding foreign antigens can be modified to coexpress cytokines to modulate immune responses toward a preferred Thelper type 1 type 2 'tilt'.

Many viruses have been proposed as live vectors. So far, the most experience in humans and nonhuman primates has been gained using poxvirus (such as modified vaccinia ankara and fowlpox) and adenovirus, vectors that can carry multiple foreign genes. Viral vectors show promise in heterologous prime-boost regimens. Recombinant hepatitis $B$ vaccine and acellular pertussis vaccines are successful recombinant protein subunit vaccines. A general advantage of subunit vaccines is their minimal reactogenicity and high level of safety. However, this safety profile changes if an antigen (such as M protein of group A Streptococcus pyogenes) is selected that potentially cross-reacts with human tissues. In contrast with the success of whole recombinant proteins, short peptide vaccines are generally poorly immunogenic.

Genomics-based vaccine strategies are useful with pathogens that cannot be readily cultivated in vitro or that do not have broadly reactive antigens readily identifiable by other methods. Elucidation of the complete genome sequence of Neisseria meningitidis group B identified candidate group B vaccine antigens by 'reverse vaccinology' ${ }^{8}$. Potential antigens identified by computer programs were expressed in Escherichia coli as recombinant fusion proteins, purified and used to immunize animals, and the resultant sera were examined for meningococcocidal activity in the presence of complement. Of 29 antigens that elicited meningococcocidal antibodies, a few active against a broad array of group B strains are being evaluated as vaccine candidates.

Various technologies allow nonliving antigens to be delivered to antigen-presenting cells. Effective nonliving antigen delivery systems are particulate, have hydrophobic moieties that insert into cell membranes and either manifest native adjuvant activity or co-deliver adjuvants in the formulation. Examples include polylactide-polyglyocolide microspheres, liposomes, virosomes, proteosomes and virus-like particles. Some, such as proteosomes (outer membrane vesicles of group B meningococcus to which antigens can be noncovalently linked) have shown promise in nonhuman primates and in clinical trials ${ }^{9}$. One innovative delivery system consists of vaccine antigens expressed in transgenic plants that are eaten as 'edible vaccines'10.

The basic idea of DNA vaccines is as simple as it is radical. A gene encoding a putative protective antigen from a pathogen is cloned into a plasmid containing a promoter active in eukaryotic cells and, after vaccination, antigen is produced in situ. The expressed protein is processed and presented to the immune system. Antibody and cytotoxic T lymphocyte responses induced by DNA vaccination in nonhuman primates and in humans are much weaker and shorter lived than those elicited in mice.

In alphavirus RNA replicon systems, structural protein genes with normally high expression from subgenomic mRNA are replaced by one or more genes encoding foreign antigen. A potential drawback of such RNA replicons is the packaging of the replicons into virus-like particles. This is still being optimized for large-scale production and must be monitored to allow detec-

\section{BOX 2 PROVEN AND PROMISING VACCINE DEVELOPMENT STRATEGIES}

- Conjugate vaccines

- Rational attenuation of known pathogens by inactivation of specific genes

- Bacterial live vector vaccines

- Viral live vector vaccines

- Subunit vaccines

- 'Reverse vaccinology' (genomics-based vaccines)

- Nonliving antigen delivery systems (such as liposomes, proteosomes, virus-like particles, virosomes and microspheres)

- DNA vaccines and replicons

- 'Heterologous' prime-boost vaccination strategies

- Powerful but well tolerated adjuvants to enhance immune responses to vaccines

- Needle-free administration of vaccines

tion of RNA recombination and generation of propagation-competent RNA genomes. In a simpler approach based on the alphavirus replicon system, cDNA driven by eukaryotic promoters expresses selfreplicating replicon $\mathrm{RNA}^{11}$. An example is SINCP, a modified plasmid replicon that incorporates nonstructural protein gene sequences from a human 'dendritic cell-tropic' Sindbis virus. Transcription from the cytomegalovirus promoter within a mammalian cell gives rise to a Sindbis virus RNA replicon vector, which programs its own cytoplasmic RNA amplification and high expression of the heterologous gene via the alphavirus subgenomic promoter. Increased immunogenicity of Sindbis-based DNA vaccines is not only due to increased antigen production. Cells transfected with DNA replicons elaborate double-stranded RNA, which enhances immune responses by stimulating Toll-like receptor 3 (TLR3) on antigen-presenting cells and induces various cytokines. Moreover, such replicons induce caspase-dependent apoptosis of transfected cells, which can increase uptake by dendritic cells. In small animals, classical DNA vaccines and Sindbis DNA replicons can be delivered mucosally using attenuated shigella and $S$. Typhi live vectors and elicit relevant immune responses including neutralizing antibodies ${ }^{12}$.

A fundamental 'vaccinology' strategy useful for poorly immunogenic antigens in animal models is 'heterologous prime-boost'; 
Table 1 Attributes of various vaccine development strategies and methods of administration of vaccines

\begin{tabular}{|c|c|c|c|c|c|c|c|c|c|c|}
\hline & $\begin{array}{l}\text { Conjugate } \\
\text { vaccines }\end{array}$ & $\begin{array}{l}\text { Attenuated } \\
\text { live } \\
\text { vaccines }\end{array}$ & $\begin{array}{l}\text { Bacterial } \\
\text { live vector } \\
\text { vaccines }\end{array}$ & $\begin{array}{l}\text { Viral } \\
\text { live vector } \\
\text { vaccines }\end{array}$ & $\begin{array}{l}\text { Subunit } \\
\text { vaccines }\end{array}$ & $\begin{array}{l}\text { Genomic- } \\
\text { based } \\
\text { vaccines }\end{array}$ & $\begin{array}{l}\text { Nonliving } \\
\text { antigen delivery } \\
\text { systems }\end{array}$ & $\begin{array}{c}\text { DNA } \\
\text { vaccines } \\
\text { and replicons }\end{array}$ & $\begin{array}{c}\text { 'Heterologous' } \\
\text { prime-boost } \\
\text { strategies }\end{array}$ & $\begin{array}{l}\text { New } \\
\text { adjuvants }\end{array}$ \\
\hline General clinical tolerability & High & High & High & High & High & High & High & High & High & Moderate \\
\hline $\begin{array}{l}\text { Potential transmissibility } \\
\text { to non-target subjects }\end{array}$ & No & Yes & Yes & Yes & No & No & No & No & $\begin{array}{l}\text { Yes (if live vector } \\
\text { vaccines used) }\end{array}$ & No \\
\hline $\begin{array}{l}\text { Safety concerns for } \\
\text { immunocompromised } \\
\text { subjects }\end{array}$ & No & Yes & Yes & Yes & No & No & No & No & $\begin{array}{l}\text { Yes (if live vector } \\
\text { vaccines used) }\end{array}$ & No \\
\hline $\begin{array}{l}\text { Likelihood of a single } \\
\text { dose immunization regimen }\end{array}$ & Low & High & Moderate & Moderate & Low & Low & Moderate & Low & No & Moderate \\
\hline \multicolumn{11}{|l|}{ Expected immunogenicity: } \\
\hline $\mathrm{T}_{\mathrm{H}} 1$ cytokine responses & Low & High & High & High & Low & Low/moderate & Moderate & Moderate & High & High \\
\hline CTL & None & High & High & High & Low & Low & Moderate & Moderate & High & High \\
\hline \multicolumn{11}{|c|}{ Potential for needle-free administration: } \\
\hline Transcutaneous & Medium & Low $^{a}$ & Low $^{a}$ & High & High & High & High & High & Moderate & Moderate \\
\hline $\begin{array}{l}\text { Needle-free } \\
\text { injection devices }\end{array}$ & High & Moderate ${ }^{a}$ & Low $^{a}$ & High & High & High & High & High & High & High \\
\hline
\end{tabular}

aPossible for live viral vaccines that are well tolerated when administered parenterally. Many bacterial vaccines (such as $S$. Typhi live vectors) are likely to be reactogenic when administered this way. $T_{H} 1, T$ helper type $1 ; C T L$, cytotoxic $T$ lymphocyte.

that is, the administration of two different vaccines that 'encode' the same antigen at various time points by the same or alternative routes. For example, when animals are primed with parenteral DNA vaccine and then boosted parenterally with a live vector expressing the relevant antigen, the immune responses and level of protection elicited are far superior to what may be achieved with other regimens. The efficacy of the DNA prime-live vector boost regimen markedly exceeds that achieved when either DNA or live vector is used for both prime and boost or when protein is used to boost after priming with DNA. Although experience with early generations of DNA vaccines in nonhuman primates and humans has been disappointing compared with their exceptional immunogenicity in small animals such as mice, DNA vaccines are nevertheless 'adept' at priming the immune systems of neonates and very young infants in the presence of maternal antibodies. This can be followed by boosting with the antigen presented in another way. Prime-boost strategies for the immunization of human infants against certain pathogens are worth pursuing, using DNA vaccines to prime. Also attractive is the strategy of mucosal priming with a vaccine antigen (such as a bacterial live vector) followed by parenteral boosting.

Adjuvants such as alum are licensed in conjunction with a specific vaccine. For years the search for useful adjuvants to enhance immune responses to vaccines was mostly empiric, and only one new adjuvant (MF59 with influenza vaccine) has been licensed in Europe and none in the US over the past four decades. Other potent candidate adjuvants tended to be reactogenic. The panoply of promising adjuvants under study is beyond the scope of this commentary. However, it should be appreciated that potent adjuvants enhance the immune response to vaccines by stimulating the innate immune system. Although overstimulation can cause clinical adverse reactions such as fever and malaise, an adjuvant that strikes the right balance will enhance immune responses yet be well tolerated. The innate immune system is activated by stimulating pattern-recognition receptors that recognize invariant molecules present in microbes but not in the host. TLRs, a family of pattern-recognition receptors, are pivotal in innate immune recognition. The modern approach to adjuvants considers what TLRs and other pattern-recognition receptors should be stimulated to achieve the desired enhanced immune response. For example, to be effective against intracellular pathogens, priming immunization should be skewed toward T helper type 1 responses. A bacterial live vector such as $S$. Typhi stimulates multiple TLRs: lipopolysaccharide stimulates TLR4; flagella stimulate TLR5; and unmethylated CpG motifs stimulate TLR9 (ref. 13).

To avoid needle and syringe, vaccines can be administered mucosally or transcutaneously or with a needle-free injection device ${ }^{7}$. Inductive sites along the gastrointestinal-associated lymphoid tissues, bronchus-associated lymphoid tissue and nasal-associated lymphoid tissue allow uptake of vaccine antigens by microfold (M) cells and transfer to underlying antigen-presenting cells including dendritic cells, macrophages and B cells.
Some live bacterial and viral vaccines (such as attenuated $S$. Typhi and shigella) that specifically target $M$ cells after mucosal administration represent a promising vaccine development strategy.

Uptake of nonliving antigens by $\mathrm{M}$ cells is less competent. Nevertheless, when nonliving antigens usually administered parenterally are given mucosally along with powerful adjuvants, robust systemic as well as mucosal responses can ensue. E. coli heat-labile enterotoxin and cholera toxin and nontoxic mutants of these molecules are strong mucosal adjuvants. However, safety questions have been raised about their use intranasally, because the ganglioside-binding property of the toxin B subunit allows these molecules to migrate centrally along olfactory nerve fibers, ultimately reaching the olfactory lobes of the brain. The new adjuvant CTA1-DD, in which cholera toxin A (ADP ribosylating) subunit is fused to a peptide (DD) that targets B lymphocytes ${ }^{14}$, offers mucosal adjuvanticity with a high level of safety. Another promising mucosal adjuvant is the polycationic polysaccharide 'chitosan' 15 .

The key to transcutaneous immunization is that the epidermis constitutes a chief immunological organ replete with Langerhans cells, a dendritic cell variant. To reach the epidermis, the keratinized stratum corneum must be made permeable by hydration, abrasion or electroporation. Antigen and adjuvant (such as E. coli heat-labile enterotoxin) applied to skin treated in this way can result in the induction of immune responses and strong effector responses ${ }^{16}$. 


\section{Concluding remarks}

Modern technologies offer rational strategies for the development of new and improved vaccines against diseases of public health importance. Regrettably, nonscientific (including financial and bioethical) impediments pose some of the most vexing barriers to the realization of the full application of these technologies. Some infectious diseases afflict mainly populations of developing countries where there are not reliable markets to ensure recuperation of investments in vaccine development. For vaccines against these diseases, what source will fund basic research and the multiple phase 1, 2 and 3 clinical trials needed over 7-12 years to bring a vaccine to licensure? Similarly, how can the complex ethical challenges be best addressed before clinical trials of vaccines are undertaken in vulnerable target populations in developing countries? The accompanying commentaries discuss these critical aspects of vaccine development. Only if acceptable solutions can be found to the financial and bioethical, as well as the technological, barriers facing vaccine development can the impending 'golden age of vaccinology' be realized.

1. Levine, M.M., Campbell, J.D. \& Kotloff, K.L. Br. Med. Bull. 62, 1-13 (2002).

2. Kaech, S.M., Wherry, E.J. \& Ahmed, R. Nat. Rev. Immunol. 2, 251-262 (2002).
3. Sprent, J. \& Surh, C.D. Annu. Rev. Immunol. 20, 551-579 (2002).

4. Esser, M.T. et al. Vaccine 21, 419-430 (2003).

5. Agematsu, K., Hokibara, S., Nagumo, H. \& Komiyama, A. Immunol. Today 21, 204-206 (2000).

6. Siegrist, C.A. Vaccine 19, 3331-3346 (2001).

7. Levine, M.M. Nat. Med. 9, 99-103 (2003).

8. Pizza, M. et al. Science 287, 1816-1820 (2000).

9. Lowell, G.H. et al. Infect. Immun. 64, 4686-4693 (1996).

10. Tacket, C.O. et al. Nat. Med. 4, 607-609 (1998).

11. Hariharan, M.J. et al. J. Virol. 72, 950-958 (1998).

12. Pasetti, M.F. et al. J. Virol. 77, 5209-5217 (2003).

13. Takeda, K., Kaisho, T. \& Akira, S. Annu. Rev. Immunol. 21, 335-376 (2003).

14. Agren, L.C., Ekman, L., Lowenadler, B., Nedrud, J.G. \& Lycke, N.Y. J. Immunol. 162, 2432-2440 (1999).

15. Mills, K.H. et al. Infect. Immun. 71, 726-732 (2003).

16. Guerena-Burgueno, F. et al. Infect. Immun. 70, 1874-1880 (2002). 